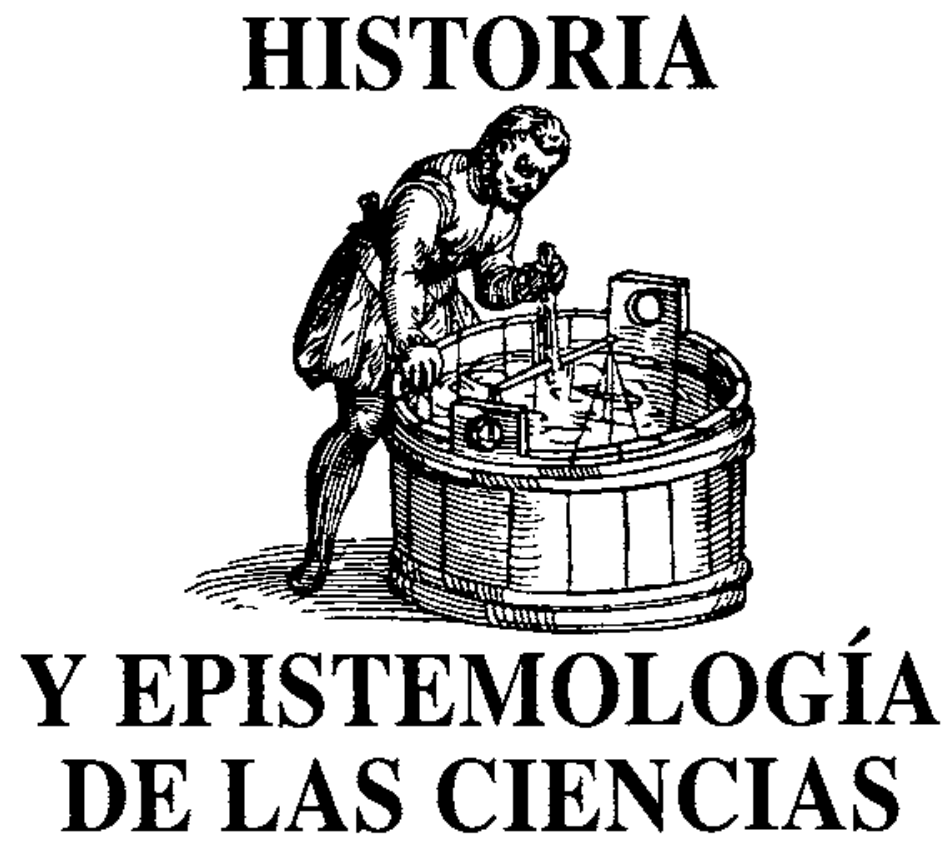

\title{
LA UTILIZACIÓN DE LA HISTORIA DE LAS CIENCIAS EN LA ENSEÑANZA DE LA FÍSICA Y LA QUÍMICA
}

SOLBES, J. ${ }^{1}$ y TRAVER, M. J. ${ }^{2}$

${ }^{1}$ CEP de València. C/ Joan de Garay, 23. 46017 València.

${ }^{2}$ Institut de Batxillerat Sant Vicent Ferrer. 46680 Algemesí.

\section{SUMMARY}

Most recently we have assisted to a considerable increase of investigations on constructivistic approaches to Science teaching, but among all these works we rarely found detailed proposals for including the History and Philosophy of Science. In this paper we consider how to initiate this trend and which should be the aims in order to get a better Science teaching. We consider basically which the pupils' perceptions of Science in a High School level are in order to show how we can modify them by means of introducing the History of Science under a new perspective.

\section{INTRODUCCIÓN}

La historia de las ciencias y sus implicaciones en la enseñanza de las mismas es una línea de investigación e innovación en educación científica con una larga tradición. Se inicia en la universidad de Harvard por Conant con el estudio, por parte de los alumnos de humanidades, de «casos» históricos, basados en el análisis de procesos clave en el desarrollo de la ciencia, con sus implicaciones filosóficas, sociales, etc. (Conant, 1957). Prosigue 
en dicha universidad con la primera edición en 1952 deI libro de Holton y en 1958 del libro de Holton y Roller, que marcaron un hito en el uso de la historia y la filosofía de la ciencia en la enseñanza de la ciencia, pero que sólo aparecen traducidos en nuestro país en fechas tan tardías como 1976 y 1963, respectivamente. Posteriormente se publicó The Project Physics Course (1970), con el mismo enfoque histórico para alumnos de ciencias de secundaria superior (16-I8 años). También hay que destacar Ios trabajos de Schwab (1962) sobre la enarrativa de la investigación», que presentan a los alumnos datos reales históricos que no pueden obtenerse en el laboratorio escolar y la descripción de las situaciones problemáticas con que se enfrentan los investigadores.

Sin embargo, en nuestro país esta línea ha influido poco en la enseñanza de las ciencias en el aula y en los materiales didácticos. Una buena prueba de este desinterés, que en el fondo revela la poca demanda por parte del profesorado, es la escasez de traducciones de libros y proyectos elaborados en esta perspectiva, así como la escasez de libros de tex to españoles, en especial pubicados por grandes editoriales, que utilizasen la historia de la ciencia. Entre las excepciones cabe destacar los textos de Beltrán y otros (1976 y 1977), el «Projecte Faraday» del grupo Recerca (1982), el libro de Calatayud y otros (1988), etc.

¿Por qué sucede esto? Cabe señalar, a tf́tulo de hipótesis, las siguientes razones. En primer lugar, la imagen de la ciencia que se transmite en la universidad a los futuros profesores, como unos contenidos ya hechos, como una serie de leyes que se deducen lógicamente a partir de unos principios. No han existido en los planes de estudio universitarios, hasta principios de los 80 , asignaturas de historia de la ciencia que pudiesen contrarrestar esa imagen y mostrasen la ciencia como una construcción de conocimientos para resolver problemas. En resumen, se presenta la ciencia pública y no la privada (Holton, 1976, Otero, 1989). Fn segundo lugar, cuando algunos profesores llegan a tener conocimientos de historia de la ciencia, en la mayoría de los casos por afición, esfuerzo personal, etc., perciben que el desarrollo de la ciencia es un proceso extraordinariamente complejo. Así, por ejemplo, es sobradamente conocido el papel que jugaron los modelos mecánicos del éter en la síntesis electromagnética de Maxwell o la importancia de las investigaciones sobre el espectro continuo de los sólidos incandescentes de Rayleigh y Jeans y, sobretodo, de Planck, para el nacimiento de la física cuántica; pero la gran dificultad didáctica de estos aspectos (Mott, 1981) imposibilita su introducción en la enseñanza secundaria. Recientemente, algunos trabajos (Matthews, 1991 y 1994) han mostrado con detalle las dificultades que presenta Ia inclusión de la historia y la filosofía de la ciencia en la enseñanza de las ciencias y han señalado argumentos a su favor, así como su inclusión en los programas de formación del profesorado de ciencias. Pero gran parte de los trabajos realizados son bastante teóricos y no muestran datos referidos a la investigación en el aula en donde se detecten las posibles carencias del alumnado, no ofrecen alternativas ni presentan innovaciones. Como ya hemos señalado anteriormente, también son muy escasos los materiales de aula que permitirían realizar la transposición didáctica de estas reflexiones tcóricas.

En nuestra propuesta no se trata de ucontar» la historia de los problemas abordados, para lo cual habría que introducir una asignatura especiffica de «historia de Ia ciencia y la técnica», sino más bien se debería clarificar qué papeles puede jugar la historia de la ciencia en la enseñanza de la misma, de acuerdo con el modelo de enseñanza-aprendizaje de la ciencia por investigación, y elaborar materiales didácticos coherentes con la propuesta. Para ello, es necesario averiguar en primer lugar qué papel juega la historia de la ciencia en la enseñanza de la física y química y qué consecuencias tiene esto en los alumnos, tanto a nivel de imagen de la ciencia como de actitudes.

\section{HIPÓTESIS Y SU OPERATTVIZACIÓN}

Nuestra hipótesis es que, en general, se ignoran los aspectos históricos en la imagen de la física y la química que se transmite y cuando se utilizan, se introducen tergiversaciones y errores históricos. Como consecuencia de ello los alumnos tienen una imagen deformada de cómo se construyen y evolucionan los conceptos cientificos.

En la enseñanza usual de la física y la química la utilización de recursos históricos es escasa y se suele concretar en el uso explícito de algunos aspectos de la historia «interna» de la ciencia, como biografías, anécdotas o grandes inventos de la técnica, y la historia de algunos conceptos o modelos sólo en algunos capítulos; por ejemplo, la naturaleza de la laz o la estructura del átomo.

Pero, además, hay una historia para uso de los científicos o reconstrucción racional de la historia caracterizada por una serie de errores y tergiversaciones entre los que destacaríamos:

- empezar los temas con observaciones o con experimentos cruciales (por ejemplo, la relatividad con la experiencia de Michelson y Morley), según un ideal empirista de la ciencia, que ignora las situaciones problemáticas que originan las investigaciones y que pucden producir cambios en las teorías existentes;

- hacer creer que el principal motivo que impulsa la creación es de índole formal, matemática, por ejemplo, cuando se dice que hizo falta el genio de Maxwell para descubrir la inconsistencia de las ecuaciones que actualmente llevan su nombre;

- mostrar que la ciencia es obra básicamente de los grandes genios, de su talento innato (ayudado por la inspiración y el azar), olvidando su carácter colectivo, fruto del trabajo de muchos hombres y mujeres. 
Por último, se introducen errores implícitamente por omisión de algunos aspectos históricos, debido a una idea de la ciencia y del currículo caracterizada por la neutralidad y la objetividad. Así:

- No se muestra el carácter tentativo de la ciencia, los errores que aparecen en el proceso de creación que genera las nuevas ideas científicas, etc. Incluso se atribuyen algunas aportaciones a científicos anteriores, como si las teorias naciesen completas. Por ejemplo, la ecuación $F=m a$ de Euler se atribuye a Newton (Truesdell, 1975) o las ecuaciones de Maxwell a este físico, olvidando a Heaviside y Lorentz (Berkson, 1981). Tampoco se muestran las limitaciones de las teorías o los problemas pendientes de solución. Todo ello no es fruto del azar sino del ideal dogmático de la ciencia como acumulación de «verdades».

- Por la misma visión acumulativa, no se muestran las crisis de los grandes paradigmas, ni los problemas en las teorías incluidas en dichos paradigmas, que producen el cambio de conceptos, modelos, etc. (salvo en el caso, ya señalado, de los modelos de la luz o del átomo).

- Se hace caso omiso del contexto histórico global del que forman parte dichas teorías científicas.

- Fruto de esta ciencia de grandes autores es la escasa valoración y, en consecuencia, el olvido de las aportaciones a la ciencia realizadas en nuestro país.

Como consecuencia de ello los alumnos tienen una visión de la ciencia caracterizada por:

- Considerar la ciencia como descubrimiento y no como una construcción de conocimientos. (Ítems A1, A7, L3).

- El empirismo (los conocimientos científicos se forman por inducción a partir de los «datos puros», es decir, las observaciones y los experimentos. (Ítems A2, L6).

- Ignorar el papel de los problemas en el desarrollo de la ciencia y, en particular, los problemas que originaron eI desarrollo de algunas teorías importantes. (Items B5, $\mathrm{L}, 7)$.

- El formalismo (la ciencia constituida básicamente por las «fórmulas», cuya aplicación mecánica permite resolver los problemas. (Ítems AS, L8).

- Un planteamiento lineal y acumulativo del desarrollo científico que no muestra la existencia de crisis (que se traducen en la aparición de nuevos paradigmas) o de cambios conceptuales en las ideas aceptadas por los científicos. (Ítems A8, Bl, L3, L9).

- Una ciencia fruto del trabajo de unos genios (cuyas contribuciones son desconocidas en algunos casos) y no como una actividad humana colectiva, de hombres y también mujeres. (Ítems B2, B3, L1 L2, L5).

- Una imagen de las ciencias físicas alejada del contexto historico social del que forma parte. (Items $\mathrm{B} 4, \mathrm{LlO}$ ).
- Se ignoran las contribuciones a la ciencia de nuestro país, con lo cual ésta parece una actividad realizada más allá de nuestras fronteras. (Ítem B2 por omisión).

\section{DISEÑO EXPERIMENTAL}

Para verificar estas hipótesis operativas se utilizarán los siguientes instrumentos:

1) En primer lugar un cuestionario (L) aplicado a diferentes libros de tex to de los que se usan mayoritariamente en las aulas en los niveles de BUP y COU para constatar el escaso papel dado a la historia de la ciencia y la presencia destacada de numerosos errores y tergiversaciones como los que ya hemos apuntado. El cuestionario se ha aplicado a modo de prueba, pero se ampliará a una muestra más extensa que complete su validez. Se han analizado los aspectos siguientes:

\section{CUESTIONARIO «L» (Libros de texto)}

L1. ¿Aparecen biografías más o menos detalladas de científicos y científicas?

L2. ¿Aparecen breves referencias marginales sobre aspectos biográficos o simples anécdotas?

L3. ¿Presentan el desarrollo histórico de algunos conceptos y teớas científicas?

LA. ¿Aparecen citas textuales de autores científicos?

L5. ¿Presentan la ciencia como obra sólo de grandes genios y no como obra colectiva de hombres y también de mujeres?

L6. ¿Contienen errores implícitos de tipo empirista o inductivista?

L7. ¿No muestran el carácter tentativo de toda investigación científica?

L8. ¿Presentan unenfoque básicamente formalista destacandio el desarrollo matemático y la aplicación de fórmulas?

L9. ¿Presentan una visión únicamente acumulativa del desarrollo de la ciencia sin destacar la aparicion de grandes crisis en los paradigmas científicos?

L10. ¿Ofrecen una vision historica y socialmente descontextualizada de los principales trabajos cientificos?

L11. ¿Proponen actividades explícitas de uso de la historia como trabajo para los alumnos y alumnas?

En los resultados, que se presentan más adelante, mostramos la importancia que se concede a cada uno de estos aspectos contabilizando el porcentaje de capítulos en que aparecen o las páginas que se dedican a cada cuestión.

2) Por otra parte se han propuesto dos cuestionarios a alumnos de BUP y COU (de 15 a 17 años) en donde se explicitan las visiones deformadas de la ciencia que Ilegan a ellos a través de la actividad docente habitual 
que muestra las carencias ya senaladas anteriormente respecto a la introducción de aspectos de historia de la ciencia. Hl primer cuestionario (A) consiste en valorar de 0 a 10 su grado, de acuerdo con las afirmaciones siguientes, que recogen algunos de los aspectos deficientes que queremos detectar.

\section{CUESTIONARIO «A» (Alumnos)}

Al. El trabajo de los físicos y los químicos consiste en descubrir las leyes físicas ocultas en la naturaleza.

A2. El método científico se puede esquematizar en el siguiente proceso: observación, experimentación, enunciacion de leyes y teorías, comprobación de las leyes y teorías enunciadas.

A3. A menudo los descubrimientos científicos se han producido por casualidad o azar.

A4. Los conceptos y modelos científicos son fieles reflejos de la realidad.

A5. El objetivo del trabajo científico es el establecimiento de relaciones matemáticas (leyes) entre magnitudes físicas.

A6. El concepto de fuerza y las leyes de la dinámica fueron establecidas por Newton en su forma actual (o no han experimentado cambios desde que fueron establecidas por Newton hasta la actualidad).

A7. Los conceptos o magnittdes que se utilizan en física y química son descubrimientos de cosas que ya existen en la naturaleza, no invenciones de los científicos.

A8. Fl desarrollo de la ciencias a lo largo de la historia ha sido un proceso acumulativo de más y más conocimientos.

En un segundo cuestionario (B) se pide de forma explícita que se contesten los siguientes aspectos.

\section{CUESTIONARIO «B» (Alumnos)}

B1. Indica brevemente las crisis que se han producido a 10 largo de la historia en el desarrollo de las ciencias físicas.

B2. Cita el nombre de al menos cinco cientificos o científicas importantes (físicos-as, químicos-as, etc.) e indica cuál fue su principal contribución al desarrollo de las ciencias físico-químicas.

B3. Indica quienes son los autores o autoras de estas contribuciones al desarrollo de la ciencia: a) ley de los gases perfectos; $b$ ) inducción electromagnética; c) aislamiento del elemento radiactivo radio; d) primer modelo cuántico del átomo; e) modelo corpuscular clásico de la luz.

B4. Citabrevemente qué repercusiones tuvieron en la sociedad en su momento histórico los siguientes desarroltos de las ciencias físico-químicas: a) modelo heliocéntrico del universo; $b$ ) inducción electromagnética; $c$ ) modelo nuclear del átomo; d) obtención de nuevos metales; e) síntesis de la urea.

B5. Trata de formular los problemas que estuvicron en el origen de los conceptos o teorias indicadas: a) teoría de la combustión de Lavoisier; $b$ ) experiencia de Oersted; c) identificación de substancias radiactivas; d) equivalencia entre calor y trabajo; e) modelo atómico de Bohr.

\section{PRESENTACIÓN Y ANÁLISIS DE RESUL- TADOS}

Seguidamente ofrecemos una valoración de los resultados obtenidos. En primer lugar una referencia global a los aspectos históricos que aparecen en los libros de texto habituales. De una muestra más amplia que también someteremos a examen, hemos seleccionado 13 libros de física y química, entre los de mayor difusión, de los niveles $2^{\circ}$ y $3^{\circ}$ de BUP y de COU. La muestra contenía un total de 319 capítulos y 5.152 páginas. El cómputo de capítulos y páginas en que aparecen referencias a aspectos históricos muestra una presencia casi siempre testimonia], pero a la vez creciente en las ediciones más recientes, de aspectos biográficos más detallados (L1: $22,6 \%$ de capítulos, pero 1,6\% de páginas) y también de referencias marginales y anecdóticas (L2: $18,2 \%$ de capítulos y $1,5 \%$ de páginas). Como cabría esperar, también es significativa la presencia del desarrollo histórico de determinados conceptos (L 3: 19,4\% de capítulos, $1,9 \%$ de páginas) y casi siempre de los que es tradicional citar: modelos atómicos, alguna controversia histórica como la dualidad de la luz o la construcción de la teoría del calor, y también los orígenes de la dinámica con Galileo y Newton.

En cambio es importante destacar la escasa presencia de materiales propiamente históricos, como citas textuales (L4: 3, I\% de capítulos, $0,2 \%$ de páginas) frente a la llamativa presencia de abundantes enfoques erróneos de tipo inductivista o empirista (L6: $17,9 \%$ de capítulos; $1,4 \%$ de páginas). Al mismo tiempo, y siendo más difícil de cuantificar, aparecen numerosos aspectos globalmente implícitos (L5, L7, L8, L9, B4), prácticamente en el $100 \%$ de los textos analizados y en ningún caso destaca el uso de la historia de la ciencia como referente didáctico ni mucho menos como posible hilo conductor.

Por último, cabe mencionar la escasa presencia de actividades explícitas de uso de la historia de la ciencia propuestas para el trabajo personal o en clase de los alumnos (LII: 16,3\% de capítulos, pero 1,3\% de páginas), aunque una vez más se hace notar su presencia creciente en ediciones más recientes, sobretodo desde principios de los 90 . No obstante, en casi todos los casos se trata de propuestas de material complementario, al final de un capítulo o sección, cuyo uso efectivo en clase podemos poner en duda en términos generales y que, por tanto, apenas liegará al alumnado más especialmente motivado para ampliar sus conocimientos.

\section{Análisis de resultados de los cuestionarios de alumnos}

Primero examinaremos las respuestas dadas al cuestionario «A» de preguntas a valorar de forma cerrada con una estimación del grado de acuerdo (de 0 a 10). Este cuestionario fue propuesto a 479 alumnos entre 15 y 17 años, de los niveles $2^{\circ}$ y $3^{\circ}$ de BUP y de COU y contiene información recogida en diferentes momentos del curso escolar, al principio del mismo y hacia la mitad, aunque no se detectan diferencias importantes entre ambas muestras. 
Al, El trabajo de los físicos y los químicos consiste en descubrir las loyes físicas ocultas en la naturaleza.

\begin{tabular}{cccc} 
Grado de acuerdo & $\begin{array}{c}0-3 \\
\text { Desacuerdo }\end{array}$ & $\begin{array}{c}4-6 \\
\text { Dudoso }\end{array}$ & $\begin{array}{c}7-10 \\
\text { De acuerdo }\end{array}$ \\
\hline Alumnos de $2^{\circ}(\mathrm{N}=209)$ & $6,7 \%$ & $28,7 \%$ & $64,6 \%$ \\
\hline Alumnos de $3^{\circ}(\mathrm{N}=134)$ & $9,0 \%$ & $32,8 \%$ & $58,2 \%$ \\
\hline Alumnos de COU $(\mathrm{N}=136)$ & $16,2 \%$ & $35,3 \%$ & $48,5 \%$ \\
\hline Tolal de alumnos $(\mathrm{N}=479)$ & $10,0 \%$ & $31,7 \%$ & $58,2 \%$ \\
\hline
\end{tabular}

El resultado de este primer ítem (Al) responde a las expectativas que teníamos, pues, aunque se observan algunas diferencias entre niveles, el conjunto de alumnos se muestra claramente de acuerdo con que la actividad científica consiste en descubrir leyes ocultas más que crear ideas y conceptos nuevos. El 58,2\% de alumnos valora con más de 6 su grado de acuerdo, frente a sólo un $10 \%$, que se muestra claramente en desacuerdo. Así pues, esta imagen de la ciencia como descubrimiento y no como construcción de conocimientos no mejora substancialmente pese a variar un poco al aumentar de nivel.

A2. El método científico se puede esquematizar en el siguiente proceso: observacion, experimentación, enunciación de leyes y teorías, comprobación de las leyes y teorías enunciadas.

\begin{tabular}{lccc} 
Grado de acuerdo & $\begin{array}{c}0.3 \\
\text { Desacuerdo }\end{array}$ & $\begin{array}{c}4-6 \\
\text { Dudoso }\end{array}$ & $\begin{array}{c}7 \cdot 10 \\
\text { De acuerdo }\end{array}$ \\
\hline Alumnos de $2^{\circ}(\mathrm{N}=209)$ & $9,6 \%$ & $9,1 \%$ & $81,3 \%$ \\
\hline Alumnos de $3^{\circ}(\mathrm{N}=134)$ & $12,7 \%$ & $12,7 \%$ & $74,6 \%$ \\
\hline Alumnos de $\operatorname{COU}(\mathrm{N}=136)$ & $14,0 \%$ & $18,4 \%$ & $67,6 \%$ \\
\hline Total de alumnos $(\mathrm{N}=479)$ & $11,7 \%$ & $12,7 \%$ & $75,6 \%$ \\
\hline
\end{tabular}

Las respuestas a este segundo ítem (A2) también están de acuerdo con nuestra hipótesis. La mayoría de alumnos $(75,6 \%)$ se muestra muy de acuerdo con un esquema en donde no aparece el carácter tentativo de la ciencia, ni la formulación de problemas, ni el enunciado de hipótesis. Apenas un $11,7 \%$ se muestra en desacuerdo. Por otra parte, esta apreciación es coherente con la imagen que transmiten la mayoría de libros de texto, que presentan esquemas semejantes sin profundizar en sus aspectos más conflictivos y sobretodo que muestran en numerosas ocasiones implícitamente un empirismo inductivista, como ya señalábamos en el ítem L6.

A3. A menudo los descubrimientos científicos se han producido por casualidad o azar.

\begin{tabular}{lccc} 
Grado de acuerdo & $\begin{array}{c}0-3 \\
\text { Desacuerdo }\end{array}$ & $\begin{array}{c}4-6 \\
\text { Dudoso }\end{array}$ & $\begin{array}{c}7.10 \\
\text { De acuerdo }\end{array}$ \\
\hline Alumnos de $2^{\circ}(\mathrm{N}=209)$ & $41,6 \%$ & $23,9 \%$ & $34,4 \%$ \\
\hline Alumnos de $3^{\circ}(\mathrm{N}=134)$ & $41,0 \%$ & $21,6 \%$ & $37,3 \%$ \\
\hline Alumnos de COU $(\mathrm{N}=136)$ & $25,7 \%$ & $39,0 \%$ & $35,3 \%$ \\
\hline Total de alumnos $(\mathrm{N}=479)$ & $37,0 \%$ & $27,6 \%$ & $35,5 \%$ \\
\hline
\end{tabular}

Los resultados del tercer ítem (A3) resultan un poco ambiguos y, aunque la mayoria de respuestas muestra desacuerdo $(37,0 \%)$, también hay un grado de acuerdo similar $(35,5 \%)$ con la af irmacion que atribuye un papel relevante al azar en la investigación, papel que puede ser reforzado por la presencia frecuente en los tibros de anécdotas sobre descubrimientos azarosos (L2) y en general por el tratamiento superficial de estos aspectos.

A4. Los conceptos y modelos científícos son fieles reflejos de la realidad.

\begin{tabular}{lccc} 
Grado de acuerdo & $0-3$ & $4-6$ & $7-10$ \\
& Desacuerdo & Dudoso & De acuerdo \\
\hline Alumnos de $2^{\circ}(\mathrm{N}=20 \mathrm{~g})$ & $9,1 \%$ & $42,1 \%$ & $48,8 \%$ \\
\hline Alumnos de $3^{\circ}(\mathrm{N}=134)$ & $23,1 \%$ & $40,3 \%$ & $36,6 \%$ \\
\hline Alumnos de COU $(\mathrm{N}=136)$ & $36,0 \%$ & $30,9 \%$ & $33,1 \%$ \\
\hline Total de alumnos $(\mathrm{N}=479)$ & $20,7 \%$ & $38,4 \%$ & $40,9 \%$ \\
\hline
\end{tabular}

De nuevo un f́tem (A4) que muestra unos resultados mayoritariamente decantados hacia el acuerdo. Pese a la dificultad que puede presentar una correcta interpretación de esta afirmación parece claro que la mayoría de alumnos $(40,9 \%)$ se inclina a pensar que existe una realidad científica objetiva y que los conceptos que han eláborado los científicos obedecen claramente a dicha realidad previa. Aunque se trate de un aspecto quizás polémico y más relacionado con la epistemología de la ciencia, no podemos dejar de considerar la manera como es percibido por el alumnado.

A5. El objetivo del trabajo científico es el establecimiento de relaciones matemáticas (leyes) entre magnitudes físicas.

\begin{tabular}{lccc} 
Grado de actuerdo & $\begin{array}{c}0+3 \\
\text { Desacuerdo }\end{array}$ & $\begin{array}{c}4-6 \\
\text { Dudoso }\end{array}$ & $\begin{array}{c}7.10 \\
\text { De acuerdo }\end{array}$ \\
\hline Alumnos de $2^{\circ}(\mathrm{N}=209)$ & $15,8 \%$ & $41,6 \%$ & $42,6 \%$ \\
\hline Alumnos de $3^{\circ}(\mathrm{N}=134)$ & $15,7 \%$ & $44,8 \%$ & $39,6 \%$ \\
\hline Alumnos de COU $(\mathrm{N}=136)$ & $20,6 \%$ & $30,9 \%$ & $48,5 \%$ \\
\hline Total de alumnos $(\mathrm{N}=479)$ & $17,1 \%$ & $39,5 \%$ & $43,4 \%$ \\
\hline
\end{tabular}

La visión formalista y la preponderancia dada al lenguaje matemático de la ciencia, frecuentemente sin una adecuada justificación, es claramente percibida por la mayoría del alumnado que se muestra de acuerdo $(43,4 \%)$ con la afirmación del item A5, aunque hay un número importante de dudosos $(39,5 \%)$. Estas respuestas confirman nuestra hipótesis, que se ve reforzada por el enfoque mayoritariamente formalista que presentan muchos libros de texto como indicábamos en el ítem L8. 
A6. El concepto de fuerza y las leyes de la dinamica fueron establecidas por Newton en su forma actual (o no han experimentado cambios desde que fueron establecidas por Newton hasta la actualidad).

\begin{tabular}{lccc} 
Grado de acuerdo & $\begin{array}{c}0-3 \\
\text { Desacuerdo }\end{array}$ & $\begin{array}{c}4-6 \\
\text { Dudoso }\end{array}$ & $\begin{array}{c}7-30 \\
\text { De acuerdo }\end{array}$ \\
\hline Alumnos de $2^{\circ}(\mathrm{N}=209)$ & $20,6 \%$ & $32,5 \%$ & $46,9 \%$ \\
\hline Alumnos de $3^{\circ}(\mathrm{N}=134)$ & $22,4 \%$ & $36,6 \%$ & $41,0 \%$ \\
\hline Alumnos de $\mathrm{COU}(\mathrm{N}=136)$ & $31,6 \%$ & $31,6 \%$ & $36,8 \%$ \\
\hline Total de alumnes $(\mathrm{N}=479)$ & $24,2 \%$ & $33,4 \%$ & $42,4 \%$ \\
\hline
\end{tabular}

'l'ambién el resultado de este ítem (A6), de claro contenido histórico, ofrece una mayoría de respuestas de acuerdo $(42,4 \%)$ con la visión que muestra la ciencia en un estadio definitivo, aun a costa de introducir tergiversaciones históricas como la que incluye el enunciado al referirse a un solo autor e ignorar a otros científicos que contribuyeron a dar forma a las leyes tal como se presentan en la enseñanza habitual. Fl importante número de dodosos $(33,4 \%)$ se puede atribuir a una ignorancia sobre dicho aspecto que en general no aparece en los libros de texto caracterizados también por una visión acabada de los conceptos científicos como mostraba el ítem 1.7 .

A7. Los conceptos o magnitudes que se utilizan en física y química son descubrimientos de cosas que ya cxisten en la naturaleza, no invenciones de los científicos.

\begin{tabular}{lccc} 
Grado de acuerdo & $\begin{array}{c}0-3 \\
\text { Desacuerdo }\end{array}$ & $\begin{array}{c}4-6 \\
\text { Dudoso }\end{array}$ & $\begin{array}{c}7.10 \\
\text { De acuerdo }\end{array}$ \\
\hline Alumnos de $2^{\circ}(\mathrm{N}=209)$ & $27,3 \%$ & $22,5 \%$ & $50,2 \%$ \\
\hline Alumnos de $3^{\circ}(\mathrm{N}=134)$ & $32,1 \%$ & $17,9 \%$ & $50,0 \%$ \\
\hline Alumnos de COC $(\mathrm{N}=136)$ & $33,8 \%$ & $18,4 \%$ & $47,8 \%$ \\
\hline Total de alumnos $(\mathrm{N}=479)$ & $30,5 \%$ & $20,0 \%$ & $49,5 \%$ \\
\hline
\end{tabular}

El ítem $\mathrm{A} 7$ refuerza los resultados del ítem $\mathrm{Al}$ sobre el trabajo científico como descubrimiento y no como creación, en este caso referido a un aspecto muy concreto como son las magnitudes físicas o los conceptos básicos. Así, una clara mayoría $(49,5 \%)$ manifiesta su acuerdo con este enunciado.

A8. El desarrollo de la ciencias a lo largo de la historia ha sido un process acumulativo de más y más conocimientos

\begin{tabular}{lccc} 
Grado de acuerdo & $0-3$ & $4-6$ & $7-10$ \\
& Desacuerdo & $\begin{array}{c}40 d o s \\
\text { Dudo acuerdo }\end{array}$ \\
\hline Alumnos de $2^{\circ}(\mathrm{N}=209)$ & $1,0 \%$ & $11,5 \%$ & $87,6 \%$ \\
\hline Alumnos de $3^{\circ}(\mathrm{N}=1,34)$ & $1,5 \%$ & $6,0 \%$ & $92,5 \%$ \\
\hline Alumnos de $\mathrm{COU}(\mathrm{N}=136)$ & $10,3 \%$ & $8,8 \%$ & $80,9 \%$ \\
\hline Total de alumnos $(\mathrm{N}=479)$ & $3,8 \%$ & $9,2 \%$ & $87,1 \%$ \\
\hline
\end{tabular}

Los resultados de este último ítem $(\Lambda 8)$ son claramente reveladores del papel preponderantemente dado al carácter acumulativo en la cvolución de la ciencia. Una mayoría muy destacada $(87,1 \%)$ muestra su acuerdo con dicha visión y no se cuestiona la aparición de grandes crisis conceptuales, como se mostrará en el siguiente cuestionario. Aun siendo cierto que en determinadas épocas florecientes de la historia de la ciencia se produce una acumulación creciente de conocimientos, los modelos científicos no son definitivos, se van produciendo cambios a distintos niveles, a modo de micro revoluciones (Toulmin, 1977), hasta que en determinados momentos se hace necesario revisar de forma drástica los esquemas conceptuales. Esto es to que algunos fikosofos de la ciencia (Kuhn, 1971) han llamado crisis de los paradigmas conceptuales o verdaderas revoluciones científicas, como el abandono de la física preclásica o aristotélica y su sustitución por la nueva física newtoniana - la superación de ésta por la física moderna o cuántica.

Finalmente examinaremos las respuestas dadas por los mismos grupos de ajumnos al cuestionario B, que en muchos aspectos corrobora nuestras hipotesis al confirmar los resultados de los cuestionarios anteriores.

BI. Indica brevemente las crisis que se han producido a lo largo de la historia en el desarrollo de las ciencias físicas.

\begin{tabular}{lcc} 
Respuestas & $\begin{array}{c}\text { Citan alguna } \\
(\%)\end{array}$ & $\begin{array}{c}\text { No citan ninguna } \\
(\%)\end{array}$ \\
\hline Alumnos de $2^{\circ}(\mathrm{N}=209)$ & 9,6 & 90,4 \\
\hline Alumnos de $3^{\circ}(\mathrm{N}=134)$ & 15,7 & 84,3 \\
\hline Alumnos de $\mathrm{COU}(\mathrm{N}=136)$ & 25,0 & 75,0 \\
\hline Total de alumnos $(\mathrm{N}=479)$ & 15,7 & 84,3 \\
\hline
\end{tabular}

Tal como mostraban los resultados del ítem A8, la mayoría de alumnos tiene una visión acumulativa de la evolución de los conocimientos científicos $\mathrm{y}$, al pedirles en este ítem (Bl) que expliciten las crisis que se han producido a lo largo de la historia de las ciencias físicas, apenas un $15,7 \%$ en conjunto es capaz de citar correctamente alguna de ellas, si bien el nivel superior da un porcentaje algo mayor $(25 \%$ en COU). También podemos señalar el notable desconcierto que genera esta cuestión, pues la mayoría responde en blanco y muchas respuestas confunden las crisis en la evolución de los conceptos con otras crisis de índole social, lo que revela quizás la incomprensión del enunciado, fiel reflejo de la ausencia de tratamiento de dicho aspecto en la enseñan7.a recibida, ya que las respuestas correctas revelan que algunos alumnos han percibido adecuadamente a qué se refería el ítem. 
B2. Citael nombre de al menos cincocientíficos ocientíficas importantes (físicos/as, químicos/as, etc.) e indica cuál fue su principal contribución al desarrolto de las ciencias fisico-quimicas.

\begin{tabular}{lccc}
$\begin{array}{l}\text { Nombres y trabajos } \\
\text { citados } \\
\text { correctamente: }\end{array}$ & So más & Menos de 5 & Ninguno \\
\hline Alumnos de $2^{\circ}(\mathrm{N}=209)$ & 20,6 & 62,2 & 17,2 \\
\hline Alumnos de $3^{\circ}(\mathrm{N}=134)$ & 33,6 & 56,0 & 10,4 \\
\hline Alumnos de COU $(\mathrm{N}=136)$ & 36,8 & 57,3 & 5,9 \\
\hline Total de alumnos $(\mathrm{N}=479)$ & 28,8 & 59,1 & 12,1 \\
\hline
\end{tabular}

En este ítem B2 donde explícitamente había que citar nombres de científicos y científicas, con especificación de sus trabajos de referencia, vemos claramente que pese al número limitado que se pedía (al menos $5)$, la mayoría $(59,1 \%)$ cita menos de 5 nombres y trabajos correctamente y sólo el $36,8 \%$ de alumnos del nivel más elevado acierta a completar la respuesta. En este mismo ítem abierto se muestra cómo la mayoría de científicos citados son hombres y corresponden a los grandes genios que reiteradamente aparecen en los libros (Newton, Galileo, Einstein, Curie, Lavoisier...). En un análisis más detallado para una parte de la muestra (123 encuestas), podemos comentar que citan a Isaac Newton un 78,8\% de alumnos de $2^{\circ}$, un $67,5 \%$ de $3^{\circ}$ y un $66^{\circ}$ de alumnos de COU, mientras que Albert Einstein es citado por un $27 \%$ de alumnos de $2^{\circ}$, un $65 \%$ de alumnos de $3^{\circ}$ y un $56 \%$ de alumnos de COU. El caso anecdótico es que la mayoría de los científicos citados son generalmente conocidos más bien debido a su popularidad extra-académica. La única referencia femenina que aparece en muchas encuestas es Marie Curie, también conocida por su popularidad. Aunque hay una gran variedad de autores, no sorprenden los nombres y son los que podriamos encontrar en cualquier libro. Una vez más cabe destacar que masivamente se citan autores de fuera de nuestro país, si exceptuamos alguna referencia puntual a Ramón y Cajal o Miguel Servet, que no son estrictamente del área de las ciencias físicoquímicas.

B3. Indica quiénes son los autores o autoras de estas contribuciones al desarrollo de la ciencia: a) ley de los gases perfectos; b) inducción clectromagnética; c) aislamiento del elemento radiactivo radio; d) primer modelo cuántico del átomo; $e$ ) modelo corpuscular clásico de la luz.

\begin{tabular}{|c|c|c|c|}
\hline $\begin{array}{l}\text { Autores } \\
\text { cilados } \\
\text { correctamente: }\end{array}$ & $\begin{array}{c}40 \text { más } \\
\text { (\%) }\end{array}$ & $\begin{array}{c}\text { Menos de } 4 \\
(\%)\end{array}$ & $\begin{array}{l}\text { Ninguno } \\
\text { (\%) }\end{array}$ \\
\hline Alumnos de $2^{\circ}(\mathrm{N}=209)$ & 0,0 & 55,0 & 45,0 \\
\hline Alumnos de $3^{\circ}(\mathrm{N}=134)$ & 6,7 & 60,5 & 32.8 \\
\hline Alumnos de $\operatorname{COU}(\mathrm{N}=136)$ & 8,8 & 78,7 & 12,5 \\
\hline Total de alumnos $(\mathrm{N}=479)$ & 4,4 & 63,3 & 32,3 \\
\hline
\end{tabular}

Cuando en el ítem $\mathrm{B} 3$ se pide concretar los nombres de autores de ciertos trabajos, las respuestas más abundantes corresponden al $63,3 \%$ de menos de 4 aciertos, que en gran parte son una única respuesta correcta, y sorprende que el $32,3 \%$ de encuestados no acierte ninguno de ellos cuando se han escogido cinco trabajos científicos sobradamente conocidos. Es más comprensible que el $45 \%$ de alumnos de $2^{\circ}$ no acierte ninguno si tenemos en cuenta que muchas de las encuestas se pasaron a principio de curso y se hace referencia a trabajos que se abordan en los programas de física y química a lo largo de los diferentes niveles.

B4. Cita brevemente qué repercusiones tuvieron en la sociedad en su momento histórico los siguientes desarrollos de las ciencias físicoquímicas: a) modelo heliocéntrico del universo; b) inducción electromagnética; c) modelo nuclear del ślomo; d) obtención dc nuevos metales; $e$ ) síntesis de la urea.

\begin{tabular}{lccc}
$\begin{array}{l}\text { Nimero de } \\
\text { repercusiones } \\
\text { ciladas }\end{array}$ & Más de 4 & Menos de 4 & Ninguna \\
\hline Alumnos de $2^{\circ}(\mathrm{N}=209)$ & 3,8 & 27,3 & 68,9 \\
\hline Alumnos de $3^{\circ}(\mathrm{N}=134)$ & 6,7 & 56,0 & 37,3 \\
\hline Alumnos de COU $(\mathrm{N}=136)$ & 11,8 & 61,7 & 26,5 \\
\hline Total de alumnos $(\mathrm{N}=479)$ & 6,9 & 45,1 & 48,0 \\
\hline
\end{tabular}

El ítem B4, de claras referencias sociohistóricas, viene a corroborar anteriores trabajos (Solbes y Vilches, 1992) sobre relaciones ciencia-técnica-sociedad, plies la mayoría de alumnos $(48 \%)$ no es capaz de citar ninguna repercusión social de hechos clave en la evolución de la ciencia precisamente por sus consecuencias y numerosas aplicaciones. Hemos citado aspectos teóricos para hacer ver si los encuestados son capaces de asociar tales aportaciones del pensamiento científico a aspectos prácticos, puesto que citar directamente inventos o aplicaciones prácticas hubiera tenido respuestas más evidentes. Pensemos en las controversias sociales del Renacimiento por la divulgación en Europa del modelo copernicano, 0 bien las innumerables aplicaciones derivadas de los trabajos de Faraday o del modelo nuclear del átono, etc. Aun así, un $45 \%$ llega a citar menos de 4 repercusiones; la mayoría responden sólo una de ellas y son numerosos los que dejan en blanco la respuesta. Resulta, pues, confirmado el carácter más teórico de la enseñanza habitual, más bien desligada de todo contexto social y que no muestra las importantes consecuencias que han tenido para la humanidad las aportaciones del pensamiento científico. 
B5. Trata de formular los problemas que estuvieron en el origen de los conceptos o teorías indicadas: $a$ ) teoría de la combustión de Lavoisier; b) experiencia de Oersted; $c$ ) identificación de substancias radioactivas; d) equivalencia entre calor y trabajo; e) modelo atómico de Bohr.

\begin{tabular}{lccc}
$\begin{array}{l}\text { Número de } \\
\text { problemas } \\
\text { formulados: }\end{array}$ & $\begin{array}{c}\text { Más de } 4 \\
(\%)\end{array}$ & $\begin{array}{c}\text { Menos de } 4 \\
(\%)\end{array}$ & $\begin{array}{c}\text { Ninguno } \\
(\%)\end{array}$ \\
\hline $\begin{array}{l}\text { Nlumnos de 20 }(\mathrm{N}=209) \\
0,0\end{array}$ & 3,4 & 96,6 \\
\hline Alumnos de $3^{\circ}(\mathrm{N}=134)$ & 0,0 & 16,4 & 83,6 \\
\hline Alumnos de COU $(\mathrm{N}=136)$ & 0,0 & 25,7 & 74,3 \\
\hline Total de alumnos $(\mathrm{N}=479)$ & 0,0 & 13,4 & 86,6 \\
\hline
\end{tabular}

Finalmente son tambićn elocuentes los resuitados de este ítem (B5) en que se muestra una abrumadora mayoría $(86,6 \%)$ de respuestas que no son capaces de formular ni siquiera brevemente qué problemas suscitaron la aparición de importantes líneas de investigación como las propuestas en el ítem. Ninguna de las encuestas ha podido dar con 4 o 5 aciertos y la mayoría de las que enuncian alguno coinciden en citar un único trabajo como máximo. Estos resultados eran de esperar, visto el nulo tratamiento que se hace en la enserianza habitual de un enfoque fundamentado en presentar la ciencia como proceso abierto de investigación que trata de abordar problemas más o menos complejos $\mathrm{y}$, por tanto, acaba por mostrarse una ciencia ya elaborada y completa que no deja posibilidad alguna de carácter abierto o tentativo.

\section{CONCIUSIONES}

El análisis de los resultados arroja en conjunto una verificación de nuestra hipótesis, pues los diferentes cuestionarios muestran cómo los aspectos de tipo histórico están ausentes de la mayoría de libros de texto y cuando aparecen son tratados de forma superficial sin atribuirles un papel relevante.

Por su parte, los cuestionarios de los alumnos revelan cómo lá enseñanza habitual, en donde están ausentes aspectos históricos, les transmite una imagen deformada de la actividad científica, ya que la mayoría de alumnos cree que la ciencia consiste en descubrir una realidad preexistente, ignora el papel fundamental del trabajo científico como resolución de problemas mediante la investigación de hipótesis y la creación de conceptos, así como la manera de evolucionar los paradigmas conceptuales, asumiendo una visión empirista, básicamente formalista y acumulativa de la ciencia y de su crecimiento.

Asimismo podemos constatar cómo persisten muchas de estas deformaciones a lo largo de los diferentes niveles sin cambiar de manera significativa. Por ello cabe pensar que la práctica docente habitual debería modificarse en sus enfoques tradicionales hacia un tratamiento adecuado de aquellos aspectos históricos que permitan mejorar sensiblemente la imagen de la ciencia que tienen los alumnos y les acerquen más a la realidad del trabajo científico. Para esto propondremos a continuación algunas posibles soluciones que abran nuevas perspectivas.

\section{PERSPECTIVAS: PAPELES DE IA HISTORIA DE LA CIENCIA Y LAS INTERACCIONES CTS EN LA ENSENANZA DE LAS CIENCIAS}

Fruto del análisis de la complejidad de la historia de la ciencia que impide, como ya hemos señalado, presentar la historia de todos los problemas, los meandros que condujeron a su solución, etc., el trabajo debe proseguir tratando de clarificar los papeles que puede jugar la historia de la ciencia en la enseñanía de la misma, su coherencia con el modelo de enseñanza-aprendizaje de la ciencia por investigación, sus relaciones con otras líneas de investigación próximas como las interacciones CrS. Esto tiene gran importancia para la didáctica de las ciencias dado que aunque ésta ya ha integrado aspectos de epistemología de la ciencia o de psicología del aprendizaje, sólo ahora está empezando a integrar aspectos de historia o de sociología de las ciencias. Buena prueba de ello es el hecho de que basta con consultar trabajos de didáctica para estar al corriente de aspectos de epistemología o de aprendizaje, pero no ocurre así con aspectos de historia o de CTS, pues éstos aún están ausentes en un tratamiento generalizado.

Respecto a las relaciones entre historia de la ciencia y CTS, pensamos que se trata de dos campos de investigación con una amplia zona de intersección: la historia social de la ciencia 0 , en otras palabras, las relaciones CTS a lo largo de la historia. Además, es necesario integrar las aportaciones de ambas líneas de investigacín en los materiales didácticos (Solbes, 1993) si queremos evitar una imagen de la ciencia y de los cientíticos en los alumnos (y en la sociedad en general) tan deformada como la que se ha caracterizado anteriormente en éste y en otros múltiples trabajos (Solbes y Vilches, 1989, Gil, 193). Esto es convergente con la idea de que el primer requisito para enseñar bien es conocer profundamente la materia a enseñar, to que supone no sólo conocimiento de los contenidos, sino también de los aspectos metodológicos, de la historia de la ciencia, de las interacciones CTS y de desarrollos científicos recientes (Gil et al., 1991).

En lo que concierne a las interacciones CTS ya hemos mostrado cn anteriores trabajos el papel que jucgan en la enseñanza de las ciencias Por otra parte, aunque gran parte de los proyectos e investigaciones del enfoque CTS surgen al margen del constructivismo, es posible utilizarlas de forma coherente con el modelo de enseñanza por investigación (Solbes y Vilches, 1989 y 1992)

En to que respecta a la historia de la ciencia, ya hemos apuntado y explicitaremos más detalladamente a continuación, los papeles que puede jugar en la enseñanza de la física y química. 
1) Permite ser críticos con la imagen tópica de la ciencia $y$, en concreto, con tergiversaciones e interpretaciones históricas que aparecen en los textos y contribuyen a dicha imagen.

2) Aunque la idea del paralelismo entre las preconcepciones de los alumnos y las concepciones vigentes a lo largo de la historia (McDermott, 1984; Carrascosa y Gil, 1985; Hashweh, 1986) ha sido cuestionada, aún se puede extraer de la historia información sobre las dificultades de los estudiantes a partir de las resistencias, de los obstáculos que se manifiestan a lo largo de ta historia de la ciencia (Saltiel y Viennot, 1985; Driver et al., 1989).

3) Favorece la selección de contenidos fundamentales de la disciplina en función de los conceptos estructurantes para introducir nuevos conocimientos y superar obstáculos epistemológicos (Gagliardi y Giordan, 1986), aunque estos aspectos sean difícilmente traducibles en forma de hilo conductor y no se puedan explicitar en determinadas actividades.

4) Permite extraer de dicha historia los problemas significativos (Gil et al., 1991) y poner al alumno en situación de abordarlos, planteando situaciones de aprendizaje que permitan a los alumnos, en cierta medida, reconstruir los conocimientos científicos. Con ello, se pretende cvitar el erróneo planteamiento empirista que introduce los experimentos sin tener en cuenta el problema histórico que los motivó, las sucesivas hipótesis que se plantearon en su interpretación, etc.

5) Permite mostrar la existencia de grandes crisis en el desarrollo de la física y la química (de la aristélicoescolástica a la clásica y de ésta a la moderna) e incluso de cambios en el interior de un paradigma (p.e., del flogisto a la teoría de la combustión de Lavoisier, del calórico de la teoría cinética del calor, de la naturaleza corpuscular de la luz a la ondulatoria, de la acción a distancia a la teoria de campos, etc.). Se trata de introducir algunas ideas no vigentes, no sólo por mostrar el carácter tentativo de la ciencia, sino por su semejanza con las preconcepciones de los alumnos o por constituir obstáculos epistemológicos. Esto puede favorecer los cambios conceptuales de los alumnos, ajustándolos a los grandes cambios de conceptos, modelos y teorías en la ciencia (Posner et al., 1982).

6) Posibilita mostrar el carácter hipotético, tentativo de Ia ciencia y mostrar, asimismo, las iimitaciones de las tcorias, sus problemas pendientes de solución, etc. Así se presenta a los alumnos la aventura de la creación científica, evitando visiones dogmáticas. Conviene también clarificar en qué forma es acumulativa la ciencia, ya que, por una parte, la mayoría de las teorías científicas aceptadas no se han derrumbado, sino que se han desarrollado, refinado y generalizado y, por otra parte, la contribución de cada científico está basada en el trabajo de muchos otros, en la naturaleza colectiva del trabajo científico).

7) Se puede mostrar la ciencia como una construcción humana, colectiva, fruto del trabajo de muchas personas, para evitar la idea de una ciencia hecha básicamente por genios, en su mayoría hombres.

8) Permite presentar las contribuciones a la ciencia realizadas en nuestro país así como los obstáculos que se le han planteado a lo largo de la historia.

9) Permite mostrar las interacciones CTS no sólo en el presente sino a lo largo de la historia, bo que facilita la comprensión de su evolución. Así, se ha pasado de la persecución ideológico-rcligiosa a que fue sometida la ciencia (Galileo, Darwin, etc.) a la constitución de la ciencia como uno de los elementos de la ideología dominante (desde el optimismo cientifista decimonónico a la actual tecnocracia). También se ha pasado de una técnica que precede a la ciencia (p.e., la construcción de mecanismos o de máquinas mecánico-térmicas precedió a su estudio por la mecánica o la termodinámica) a una ciencia origen de múltiples aplicaciones técnicas $c$, in * cluso, de ramas de la producción (la cléctrica y la química en el siglo XIX, la electrónica en la actualidad).

10) Contribuye a mejorar las actitudes del alumnado hacia la ciencia y su aprendizaje (Izquierdo, 1994).

Después de realizar este proceso de clarificación sobre tos papeles que puede jugar la historia de la ciencia en la enseñanza de la física y la química y, por tanto, la contribución que puede realizar en la didáctica de las ciencias, el siguiente paso consiste en elaborar materiales didácticos que supongan la puesta en práctica de la propuesta, utilizarlos en el aula y comparar los resultados obtenidos por estos alumnos con los antes presentados, que servirán de grupo de control.

En concreto, nuestra propuesta metodológica consiste en elaborar y someter a análisis un amplio y variado catálogo de actividades de claro contenido y enfoque histórico para el trabajo en el aula, que supere las deficiencias ya apuntadas anteriormente, abarcando el máximo de temas habituales en los contenidos de las enseñanzas secundarias. Este catálogo se puede ampliar con la elaboración más completa de determinados amas que permiten un enfoque global ce los aspectos históricos, para mostrar que se pueden usar no solamente como material complementario sino formando parte sustancial del hilo conductor de los contenidos abordados en la enseñanza de las ciencias físico-quuímicas. 


\section{REFERENCIAS BIBLIOGRÁFICAS}

BELTRAN, J. et al. (1976), Fisica y química $2^{\circ} B U P$. Madrid: Editorial Anaya.

BELTRAN, J. et al. (1977), Física y química $3^{\circ}$ BUP. Madrid: Editorial Anaya.

BERKSON (1981). Las teorias de los campos de fuerzas. Desde Faraday hasta Einstein. Madrid: Alianza Universidad.

CALATAYUD, M.L. et al. (1988). La construcción de las ciencias físico-químicas. Servei de Formació Permanent. Universitat de València. Valencia: Nau Llibres.

CARRASCOSA, J. y GIL, D. (1985). La metodología de la superficialitat i l'aprenentatge de les ciencics. Enseñanza de las Ciencias, 3(2), pp. 113-120.

CONANT, J.B., (ed.), (1957). Harvard Case Histories in Experimental Science. 2 vols. Cambridge: Harvard University Press.

DRIVER, R. et al. (1989). Ldeas cientificas en la infancia y la adolescencia. Madrid: Morata/MEC

GAGLIARDI, R. y GIORDAN, A. (1986). La historia de las ciencias: una herramienta para la enseñanza. Enseñanza de las Ciencits: 4(3), pp. 253-259.

GIL, D. et al. (1991). La enseñanza de las ciencias en la educación secundaria. Barcelona: IC'E-Horsori.

GIL, D. (1993). Contribución de la historia y de la filosofía de las ciencias al desarrollo de un modelode enseñanza-aprendizaje como investigación. Enseñanza de las Ciencias, 11(2), pp. 197.212.

GRUP RECERCA (1982). Projecte Faraday. Física i química per al hatxillerat. Barcelona: IC.E, Universitat Autònoma.

HASHWEH, M. Z. (1986). T'owards an explanation of conceptual change. European Journal of Science Education, 8 (3), pp. 229-249.

HOLTON, G. y BRUSH, S.G. (1976). Introducción a los conceptos y teorías de las ciencias físicas. Barcelona: Reverté.

HOLTON, G. y ROLLER, D. (1963), Fundamentos de la Física Moderna. Barcelona: Reverté.

I\%QUIERDO, M. (1994). Cómo contribuye la historia de las ciencias en las actitudes del alumnado hacia la enseñanza de las ciencias. Aula de Innovación Educativa, 27, pp. 37-41.

KUHN, D. (1971), La estructura de las revoluciones cientificas. México: Fondo de Cultura Económica.

MATTHEWS, M.R. (1991). Un lugar para la historia y la fílosofía en la enseñanza de las ciencias. Comunicación. Lenguaje y Educación, 11-12, pp. 141-155.
MATTHEWS, M.R. (1994). Historia, filosofía y enseñanza de las ciencias: la aproximación actual. Enseñanzade les Ciencias, 12(2), pp. 255-277.

McDERMOTT, L.C. (1984), Research on concepual understanding in mechanics. Physics Today, Julio, pp. 24-34.

MOTT, N. (1981). On teaching quantum phenomena, en Quanttum Mechanics in the School. (International Comission of Plyssics Education of IUPAP).

OTERO, J. (1989). La producción y la comprensión de la ciencia: la elaboración en el aprendizaje de la ciencia escolar. Enseñanza de las Ciencias. 7(3), pp. 223-228.

POSNFR, G.L. et al. (1982). Accomodation of a scientific conception: Toward a theory of conceptual chantre. Science Education, 66(2), pp. 211-227.

SALTIEL, E. y VIENNOT, L, (1985). ¿Qué aprendemos de las semejanzas entre las ideas históricas y el razonamiento espontáneo de los estudiantes? Enseñanza de las Ciencias, 3(2), pp. 137-144.

SCHWAB, J. J. (1962). The Teaching of Science as Enquiry, en The Teaching of Science. Cambridge: Harvard University Press.

SOLBES, J. y VILCHES, A. (1992). El modelo constructivista y las relaciones ciencia-técnica-sociedad. Enseñan ${ }_{\star} a$ de las Ciencias, 7(1) pp. 181-186.

SOLBES, J. y VILCHES, A. (1989). Interacciones cienciatécnica-sociedad. Un instrumento de cambio actitudinal Enseñanza de las Ciencias, 7(1). pp. 14-20.

SOLBES, J. (1993). Física $2^{\circ}$ Bachillerato. Materiales didáticos. Madrid: MEC.

TOULMIN, S. (1977). La comprensión humanu (I): el uso colectivo y la evolución de los conceptos. Madrid: Alianza Editorial.

TRUESDELL (1975). Ensavos de historia do la mecanica. Madrid: Editorial Tecnos.

VARIOS (1970), The Project Physics Course. Nueva York: Holt-Rineart-Winston.

[Artículo recibido en noviembre de 1994 y aceptado en diciembre de 1995.] 